\title{
Amentoflavone enhances sorafenib-induced apoptosis through extrinsic and intrinsic pathways in sorafenib-resistant hepatocellular carcinoma SK-Hep1 cells in vitro
}

\author{
WEI-LUNG CHEN ${ }^{1,2^{*}}$, CHIA-LING HSIEH ${ }^{3 *}$, JIANN-HWA CHEN $^{1,2}$, CHIH-SHENG HUANG $^{4,5}$, \\ WEI-TING $\mathrm{CHEN}^{6}$, YU-CHENG KUO ${ }^{7}, \mathrm{CHENG}^{-} \mathrm{YU} \mathrm{CHEN}^{8,9^{*}}$ and FEI-TING $\mathrm{HSU}^{8-10}$ \\ ${ }^{1}$ Department of Emergency Medicine, Cathay General Hospital, Taipei 106; ${ }^{2}$ School of Medicine, \\ Fu-Jen Catholic University, Taipei 242; ${ }^{3}$ The Ph.D. Program for Translational Medicine College of Medical Science \\ and Technology, Taipei Medical University, Taipei 110; ${ }^{4}$ Division of Colon and Rectal Surgery, Department of \\ Surgery, National Yang-Ming University Hospital, Yilan 260; ${ }^{5}$ Department of Surgery, School of Medicine, \\ National Yang-Ming University, Taipei 112; ${ }^{6}$ Department of Psychiatry, Zuoying Branch of Kaohsiung Armed \\ Forces General Hospital, Kaohsiung 813; ${ }^{7}$ Radiation Oncology, Show Chwan Memorial Hospital, Changhua 500; \\ ${ }^{8}$ Department of Medical Imaging, Taipei Medical University Hospital; ${ }^{9}$ Translational Imaging Research Center; \\ ${ }^{10}$ Department of Radiology, School of Medicine, Taipei Medical University, Taipei 110, Taiwan, R.O.C.
}

Received March 4, 2016; Accepted April 25, 2017

DOI: $10.3892 / \mathrm{ol} .2017 .6540$

\begin{abstract}
The present study aimed to evaluate the effects of amentoflavone on sorafenib-induced apoptosis in sorafenib-resistant hepatocellular carcinoma (HCC) cells. The sorafenib-resistant SK-Hep1 (SK-Hep1R) cell line was established for the present study. Initially, the differences in sorafenib-induced cytotoxicity and apoptosis between wild-type SK-Hep1 and SK-Hep1R cells were verified using the MTT assay and flow cytometry. The effects of amentoflavone on sorafenib-induced cytotoxicity and apoptosis were then investigated using MTT, flow cytometry, DNA gel electrophoresis and western blot analysis. The results demonstrated that cell viability of SK-Hep1R cells was increased compared with that of SK-Hep1 cells following
\end{abstract}

Correspondence to: Dr Fei-Ting Hsu, Department of Medical Imaging, Taipei Medical University Hospital, 252 Wuxing Street, Taipei 110, Taiwan, R.O.C.

E-mail: sakiro920@gmail.com

*Contributed equally

Abbreviations: HCC, hepatocellular carcinoma; SK-Hep1R, SK-Hep1 sorafenib-resistant; MMP, mitochondrial membrane potential; XIAP, X-linked inhibitor of apoptosis protein; Mcl-1, myeloid cell leukemia-1; C-FLIP, cellular FADD-like IL-1 $\beta$-converting enzyme FLICE-like inhibitory protein; NF- $\kappa \mathrm{B}$, nuclear factor-kB; STAT3, signal transducer and activator of transcription 3

Key words: amentoflavone, sorafenib, resistance, apoptosis, hepatocellular carcinoma treatment with different concentrations of sorafenib for $24 \mathrm{~h}$. Apoptosis of SK-Hep1R cells was lower than that of SK-Hep1 cells following treatment with $20 \mu \mathrm{M}$ sorafenib for $24 \mathrm{~h}$. Amentoflavone alone did not inhibit cell viability but significantly triggered sorafenib-induced cytotoxicity and apoptosis in SK-Hep1R cells. Amentoflavone not only reversed sorafenib-induced anti-apoptotic protein levels but also enhanced sorafenib-induced pro-apoptotic protein expression in SK-Hep1R cells. In conclusion, amentoflavone may be used as a sorafenib sensitizer to enhance sorafenib-induced cytotoxicity and trigger sorafenib-induced apoptosis through extrinsic and intrinsic pathways in SK-Hep1R cells.

\section{Introduction}

Sorafenib, a multi-kinase inhibitor, has been approved by the US Food and Drug Administration to improve overall survival and time to progression of patients with advanced hepatocellular carcinoma (HCC) (1). Sorafenib induces apoptosis and inhibits angiogenesis in HCC through blockage of the rapidly accelerated fibrosarcoma/mitogen-activated protein kinase/extracellular signal-regulated kinase cascade, vascular endothelial growth factor and platelet-derived growth factor receptor tyrosine kinase signaling $(2,3)$. Sorafenib has also been demonstrated to enhance the therapeutic efficacy of anticancer agents and radiotherapy via inhibition of nuclear factor- $\kappa \mathrm{B}(\mathrm{NF}-\kappa \mathrm{B})$ or signal transducer and activator of transcription 3 (STAT3)-modulated resistance to anticancer treatments in $\mathrm{HCC}$ models in vitro and in vivo $(4,5)$. However, long-term exposure to sorafenib for HCC cells induces sorafenib resistance and results in tumor progression $(6,7)$. Therefore, development of sorafenib sensitizers, which reverse sorafenib resistance and results in sorafenib-inhibited tumor progression in sorafenib-resistant HCC cells, is important. 
Previous studies have identified the molecular mechanism of sorafenib resistance and have identified different types of sorafenib sensitizers. For example, Chen et al (8) reported that activation of phosphatidylinositol 3-kinase/protein kinase B (Akt) signaling modulates acquired resistance to sorafenib in HCC cells. Akt inhibitors may enhance sorafenib-induced apoptosis in HCC cells with sorafenib resistance. Tai et al (9) reported that dovitinib, a novel Src homology region 2 domain-containing phosphatase-1 (SHP-1) activator, induces apoptosis and overcomes soreafenib resistance through SHP-1-inhibited STAT3 activation in HCC cells. Cell cycle and anti-apoptosis associated proteins are overexpressed by sorafenib treatment in sorafenib-resistant HCC cells. In addition, Hsu et al (10) proposed that Cyclin-E1 and myeloid cell leukemia-1 (Mcl-1) overexpression inhibits sorafenib-induced apoptosis, whereas suppression of Cyclin-E1 and Mcl-1 enhances induction of apoptosis. Based on these previous studies, it was hypothesized that restoration of sorafenib-induced apoptosis by sorafenib sensitizers is a critical mechanism in overcoming sorafenib resistance in HCC cells.

Amentoflavone, a polyphenolic compound isolated from Selaginella tamariscina, has been demonstrated to possess anticancer effects through the inhibition of molecules that are associated with tumor progression and modulation of apoptosis (11-13). Amentoflavone, as a NF- $\kappa \mathrm{B}$ signal inhibitor, induces anti-angiogenic and anti-metastatic effects via suppression of $\mathrm{NF}-\kappa \mathrm{B}$ activation in breast cancer and melanoma cells in vitro and in vivo $(11,12)$. Amentoflavone has also been suggested to induce apoptosis and inhibit Akt phosphorylation in cervical and breast cancer cells $(14,15)$. However, whether amentoflavone, as a sorafenib sensitizer, triggers sorafenib-induced apoptosis in sorafenib-resistant HCC cells remains ambiguous. The present study aimed to investigate the effects of amentoflavone on sorafenib-induced apoptosis in sorafenib-resistant HCC cells. In the present study, sorafenib-resistant HCC SK-Hep1 (SK-Hep1R) cells were established, and were selected following long-term sorafenib exposure. Effects of sorafenib on cell viability and apoptosis were evaluated in wild-type SK-Hep1 and SK-Hep1R cells by MTT assay and flow cytometry. Effects of sorafenib, amentoflavone and a combination of the two on cell viability, apoptosis and expression of anti-apoptotic and pro-apoptotic proteins were also investigated in SK-Hep1R cells, using MTT, flow cytometry, DNA gel electrophoresis and western blot analysis.

\section{Materials and methods}

Chemicals. Sorafenib (Nexavar) was provided by Bayer Health Care Pharmaceuticals, Inc. (Whippany, NJ, USA). Dulbecco's modified Eagle's medium (DMEM), fetal bovine serum (FBS), L-glutamine and penicillin-streptomycin were bought from Gibco; Thermo Fisher Scientific, Inc. (Waltham, MA, USA). Propidium iodide (PI) and 3,3'-dihexyloxacarbocyanine iodide $\left(\mathrm{DiOC}_{6}\right)$ were purchased from BioVision, Inc. (Milpitas, CA, USA) and Enzo Life Sciences, Inc. (Farmingdale, NY, USA), respectively. MTT and RNase were obtained from Sigma-Aldrich; Merck KGaA (Darmstadt, Germany) and Fermentas; Thermo Fisher Scientific, Inc., respectively.
Primary antibodies for cleaved Caspase-3 (dilution, 1:500; catalog no.P42574; anti-rabbit) and cellular FLICE (FADD-like IL-1 $\beta$-converting enzyme)-inhibitory protein (C-FLIP) (dilution, 1:500; catalog no. O15519; anti-rabbit) were bought from Cell Signaling Technology, Inc. (Danvers, MA, USA). Primary antibodies of cleaved Caspase-8 (dilution, 1:500; catalog no. MA5-15054; anti-rabbit) and X-linked inhibitor of apoptosis protein (XIAP) (dilution, 1:500; catalog no. PA1-84846; anti-rabbit) were purchased from Thermo Fisher Scientific, Inc. Primary antibodies of Mcl-1 (dilution, 1:500; catalog no. 3035-100; anti-rabbit) and cytochrome $c$ (dilution, 1:500; catalog no. sc-13156; anti-mouse) were obtained from BioVision, Inc. and Santa Cruz Biotechnology, Inc. (Dallas, TX, USA), respectively. Horseradish peroxidase-conjugated secondary antibodies were bought from Jackson ImmunoResearch Laboratories, Inc. (catalog nos. 31430 and 31460; dilution, 1:5,000; West Grove, PA, USA). Nuclear and Cytoplasmic Extraction and Genomic DNA miniprep kits were purchased from Chemicon; EMD Millipore (Billerica, MA, USA) and Axygen; Corning Incorporated (Corning, NY, USA), respectively.

Cell culture. SK-Hep1 cells were provided by Professor Jing-Gung Chung (Department of Biological Science and Technology, China Medical University, Taichung, Taiwan). Cells were cultured in DMEM supplemented with $10 \%$ FBS, $2 \mathrm{mM}$ L-glutamine, $100 \mathrm{U} / \mathrm{ml}$ penicillin and $100 \mathrm{mg} / \mathrm{ml}$ streptomycin, and maintained in a humidified incubator at $37^{\circ} \mathrm{C}$ in an atmosphere of $5 \% \mathrm{CO}_{2}(16)$.

Establishment of sorafenib-resistant SK-Hepl cells. The sorafenib-resistant SK-Hep1 (SK-Hep1R) cells were selected from SK-Hep1 cells that survived slowly escalating concentrations of sorafenib treatment (2.5 $\mu \mathrm{M}$ increase per month) till reached $10 \mu \mathrm{M}$ was reached, as previously described by Zhai et al (17). Finally, after 3-4 month, SK-Hep1R cells were cultured in medium containing $10 \mu \mathrm{M}$ sorafenib for use in the present study.

MTT assay. SK-Hep1 or SK-Hep1R cells were seeded onto 96-well plates at a density of $3 \times 10^{4}$ cells/well and incubated overnight. SK-Hep1 and SK-Hep1R cells were treated with $0,10,15,20$ and $25 \mu \mathrm{M}$ sorafenib in $0.1 \%$ dimethyl for $24 \mathrm{~h}$. In addition, SK-Hep1R cells were treated with 0-25 $\mu \mathrm{M}$ sorafenib alone or combined with $75 \mu \mathrm{M}$ amentoflavone for $24 \mathrm{~h}$. Cell viability was evaluated by MTT assay, as described previously (4).

Detection of mitochondrial membrane potential (MMP). SK-Hep1 or SK-Hep1R cells were seeded onto 12-well plates at a density of $2 \times 10^{5}$ cells/well and incubated overnight. SK-Hep1 and SK-Hep1R cells were treated with $0 \mu \mathrm{M}$ or $20 \mu \mathrm{M}$ sorafenib in $0.1 \%$ dimethyl for $24 \mathrm{~h}$. For combination treatment, SK-Hep1R cells were treated with $20 \mu \mathrm{M}$ sorafenib, $75 \mu \mathrm{M}$ amentoflavone or a combination of these for $24 \mathrm{~h}$. Cells from different groups were harvested by centrifugation, washed twice with PBS, resuspended in $500 \mu \mathrm{l} \mathrm{PBS}$ with $4 \mu \mathrm{M}$ $\mathrm{DiOC}_{6}$ and incubated for $30 \mathrm{~min}$ at $37^{\circ} \mathrm{C}$. The changes of MMP were measured by flow cytometry (FACSCalibur FACS101; BD Biosciences, Franklin Lakes, NJ, USA) as previously 
A

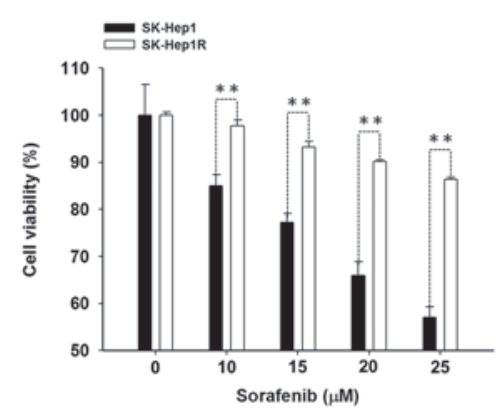

B
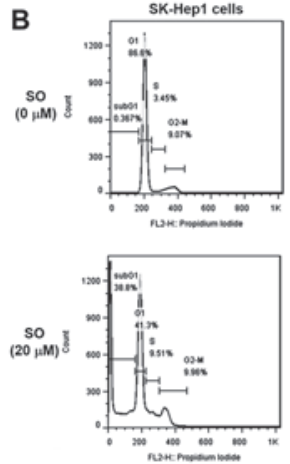
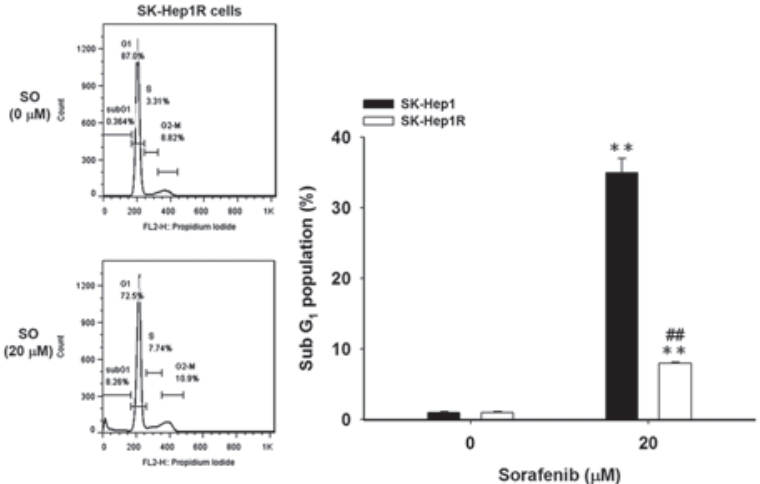

C
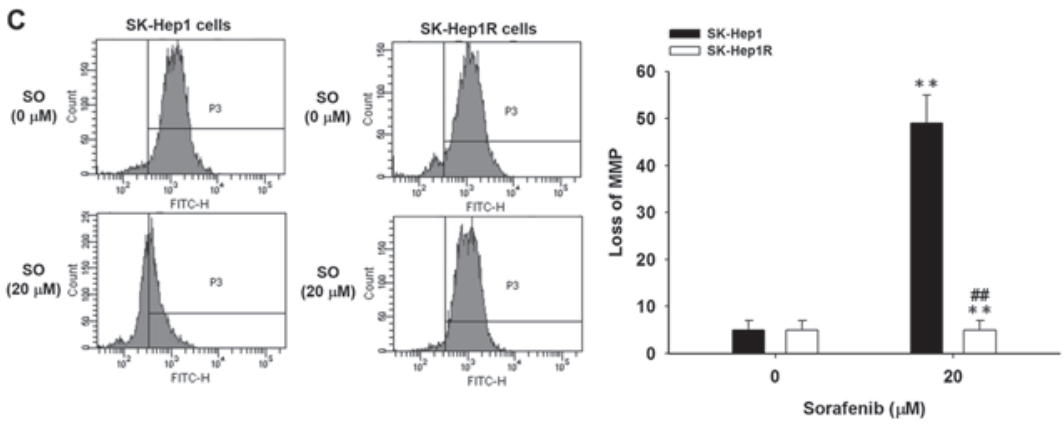

Figure 1. Effect of sorafenib-induced cytotoxicity and apoptosis on SK-Hep1 and SK-Hep1R cells. SK-Hep1 and SK-Hep1R cells were treated with 0, 10, 15, 20 and $25 \mu \mathrm{M}$ sorafenib in $0.1 \%$ dimethyl for $24 \mathrm{~h}$. (A) Cell viability was investigated by MTT assay. (B) Analysis of the subG $\mathrm{G}_{1}$ population was evaluated by flow cytometry. (C) Detection of MMP was evaluated by flow cytometry. ${ }^{* *} \mathrm{P}<0.01$ vs. control, ${ }^{\# \#} \mathrm{P}<0.01$ vs. sorafenib in SK-Hep1 cells. SK-Hep1R, SK-Hep1 sorafenib-resistant; MMP, mitochondrial membrane potential; SO, sorafenib; FITC, fluorescein isothiocyanate.

described (18). All data were analyzed by FlowJo 7.6.1 software (Tree Star, Inc., Ashland, OR, USA).

Analysis of the subG $G_{1}$ population. SK-Hep1 or SK-Hep1R cells were seeded onto 12 -well plates at a density of $2 \times 10^{5}$ cells/well and incubated overnight. SK-Hep1 and SK-Hep1R cells were treated with $0 \mu \mathrm{M}$ or $20 \mu \mathrm{M}$ sorafenib in $0.1 \%$ dimethyl for $24 \mathrm{~h}$. For combination treatment, SK-Hep1R cells were treated with $20 \mu \mathrm{M}$ sorafenib, $75 \mu \mathrm{M}$ amentoflavone or a combination of these for $24 \mathrm{~h}$. Cells were collected, fixed with $70 \%$ ethanol and incubated overnight at $-20^{\circ} \mathrm{C}$. Cells were washed with PBS and then resuspended in $500 \mu \mathrm{l}$ PI buffer $(40 \mu \mathrm{g} / \mathrm{ml}$ PI, $100 \mu \mathrm{g} / \mathrm{ml}$ RNase and $1 \%$ Triton X-100 in PBS) (catalog no. P1304MP; Thermo Fisher Scientific, Inc.) for $1 \mathrm{~h}$ in the dark at room temperature. Detection of the subG $\mathrm{G}_{1}$ population was evaluated by flow cytometry (FACSCalibur FACS101; BD Biosciences) as described by Huang et al (19). All data were analyzed by FlowJo 7.6.1 software (Tree Star, Inc.).

Detection of DNA fragmentation. SK-Hep1R cells were seeded onto 6-well plates at a density of $1 \times 10^{6}$ cells/well and incubated overnight. Cells were then treated with $20 \mu \mathrm{M}$ sorafenib, $75 \mu \mathrm{M}$ amentoflavone and their combination for $24 \mathrm{~h}$. The genomic DNA miniprep kit (Chemicon; EMD Millipore) was used to purify genomic DNA from cells, following the protocol provided by the manufacturer. Detection of DNA fragmentation was analyzed using $1.5 \%$ agarose gel electrophoresis with SYBRsafe stain (4).

Western blot analysis. A total of $3 \times 10^{6}$ SK-Hep1 or SK-Hep1R cells were seeded in $10 \mathrm{~cm}$ diameter dishes and incubated overnight. SK-Hep1 cells were treated with $20 \mu \mathrm{M}$ sorafenib for $24 \mathrm{~h}$. In addition, SK-Hep1R cells were treated with $20 \mu \mathrm{M}$ sorafenib, $75 \mu \mathrm{M}$ amentoflavone or a combination of these for $24 \mathrm{~h}$. Total proteins from cells were extracted with lysis buffer (50 mM Tris- $\mathrm{HCl}(\mathrm{pH} 8.0), 120 \mathrm{mM} \mathrm{NaCl}, 0.5 \%$ NP-40 and $1 \mathrm{mM}$ phenylmethanesulfonyl fluoride). A cytosol extraction kit (catalog no. 2118936; EMD Millipore) was used to extract cytosolic cytochrome $c$ from cells, following the protocol provided by the manufacturer. Expression levels of XIAP, Mcl-1, C-FLIP, Capase-3, Caspase-8 and cytochrome $c$ were determined by western blot analysis, as described by Ting et al (20). The levels of protein bands were quantified with Image J software version 1.48 (National Institutes of Health, Bethesda, MD, USA).

Statistical analysis. All data are presented as the mean \pm standard error. Student's t-test was analyzed for comparison between the control and each treatment group by SigmaPlot version 10 (Systat Software, Inc., San Jose, CA, USA). P<0.05 was considered to indicate a statistically significant difference.

\section{Results}

Differences in sorafenib-induced cytotoxicity and apoptosis between SK-Hepl and sorafenib-resistant SK-Hepl cells. Differences in sorafenib-induced cytotoxicity were examined between SK-Hep1 and SK-Hep1R cells using the MTT assay. The viability of SK-Hep1R cells was significantly increased compared with viability of wild-type SK-Hep1 cells following treatment with 10-25 $\mu \mathrm{M}$ sorafenib for $24 \mathrm{~h}$ (Fig. 1A). Sorafenib treatment $(10-25 \mu \mathrm{M})$ significantly reduced cell viability by 
A

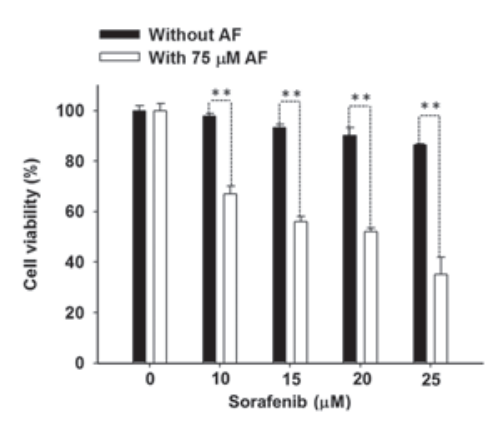

C

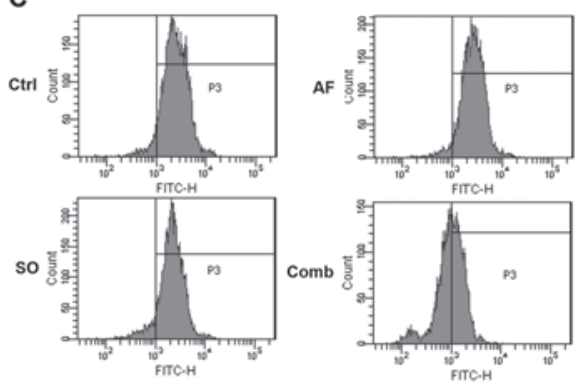

B
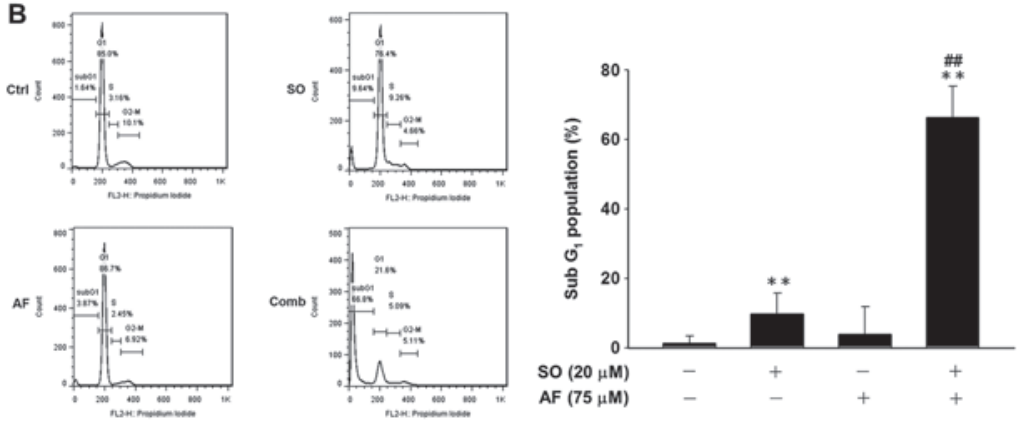

D

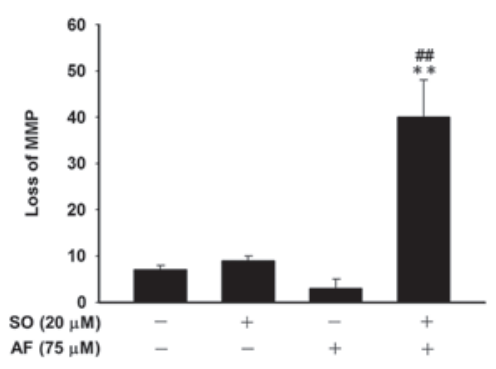

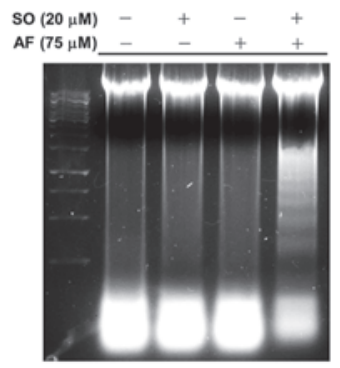

Figure 2. Effect of amentoflavone on sorafenib-induced cytotoxicity and apoptosis in SK-Hep1R cells. SK-Hep1R cells were treated with 0-25 $\mu \mathrm{M}$ sorafenib alone or combined with $75 \mu \mathrm{M}$ amentoflavone for $24 \mathrm{~h}$ in SK-Hep1R cells. (A) Cell viability was investigated by MTT assay. (B) Analysis of the subG $\mathrm{M}_{1}$ population was evaluated by flow cytometry. (C) Measurement of MMP was evaluated by flow cytometry. (D) Detection of DNA fragmentation was investigated with DNA gel electrophoresis. ${ }^{* *} \mathrm{P}<0.01$ vs. control, ${ }^{\# \#} \mathrm{P}<0.01$ vs. sorafeninb alone. SK-Hep1R, SK-Hep1 sorafenib-resistant; MMP, mitochondrial membrane potential; Ctrl, control; AF, amentoflavone; SO, sorafenib; Comb, combination; FITC, fluorescein isothiocyanate.

$15-46 \%$ compared with the control SK-Hep1 cells. Notably, no evident cytotoxicity was observed when SK-Hep1R cells were treated with $10 \mu \mathrm{M}$ sorafenib for $24 \mathrm{~h}$. Sorafenib treatment $(15-25 \mu \mathrm{M})$ significantly reduced cell viability by $7-14 \%$ compared with that of the control in SK-Hep1R cells. Differences in sorafenib-induced apoptosis between SK-Hep1 and SK-Hep1R cells were investigated by detection of subG $_{1}$ and MMP with flow cytometry. The subG $\mathrm{G}_{1}$ population of SK-Hep1R cells was significantly decreased compared with wild-type SK-Hep1 cells following treatment with $20 \mu \mathrm{M}$ sorafenib for $24 \mathrm{~h}$. Sorafenib significantly increased the $\mathrm{subG}_{1}$ population by $35 \%$ compared with the control SK-Hep1 cells, and only increased subG $_{1}$ population by $8 \%$ compared with the control SK-Hep1R cells (Fig. 1B). SK-Hep1R cells were also demonstrated to present resistance to sorafenib-induced loss of MMP. Sorafenib treatment $(20 \mu \mathrm{M})$ significantly reduced MMP by $50 \%$ compared with the control SK-Hep1 cells (Fig. 1C). In contrast, the MMP of SK-Hep1R cells was not affected under similar experimental conditions.

Amentoflavone triggers sorafenib-induced cytotoxicity and apoptosis in sorafenib-resistant SK-Hepl cells. Cytotoxicity in SK-Hep1R cells was significantly increased following combined treatment compared with sorafenib alone (Fig. 2A). Combinational treatment and sorafenib alone significantly increased the subG ${ }_{1}$ population by 66 and $9.7 \%$ compared with the control, respectively (Fig. 2B). A combination of amentoflavone and sorafenib significantly increased the loss of MMP compared with other treatment groups in SK-Hep1R cells (Fig. 2C). Combined treatment was also demonstrated to induce visible DNA fragmentation (Fig. 2D).
Amentoflavone restores sorafenib-induced apoptosis in extrinsic and intrinsic pathways in sorafenib-resistant SK-Hepl cells. The levels of anti-apoptotic proteins (XIAP, Mcl-1 and C-FLIP) were reduced by 0.7-0.8 fold in SK-Hep1 cells compared with SK-Hep1R cells following treatment with $20 \mu \mathrm{M}$ sorafenib for $24 \mathrm{~h}$, but anti-apoptotic protein levels of SK-Hep1R cells were not inhibited under similar experimental conditions (Fig. 3A and B). Amentoflavone not only inhibited sorafenib-induced anti-apoptotic protein levels (XIAP, Mcl-1 and C-FLIP) but also triggered sorafenib-induced pro-apoptotic protein expression (cleaved-Caspase-3, -8 and cytochrome $c$ ) in SK-Hep1R cells (Fig. 3B).

\section{Discussion}

Sorafenib is the only FDA approved drug for advanced $\mathrm{HCC}$, but acquired resistance limits the therapeutic efficacy of sorafenib. Therefore, development of sorafenib sensitizers may benefit patients with HCC. Based on selected published studies, it was hypothesized that restoration of sorafenib-induced apoptosis by sensitizers is critical in overcoming acquired sorafenib resistance in HCC cells. Amentoflavone has been demonstrated to inhibit tumor growth through induction of apoptosis in breast and cervical cancer cells $(14,15)$. However, whether amentoflavone is able to act as a sorafenib sensitizer, which restores sorafenib-induced apoptosis in sorafenib-resistant HCC cells, has not been elucidated. The present study aimed to evaluate the effect of amentoflavone on sorafenib-induced apoptosis in sorafenib-resistant HCC cells. A sorafenib-resistant SK-Hep1 cell line was established and used in the present 
A

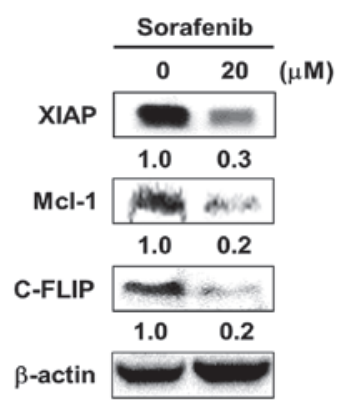

B

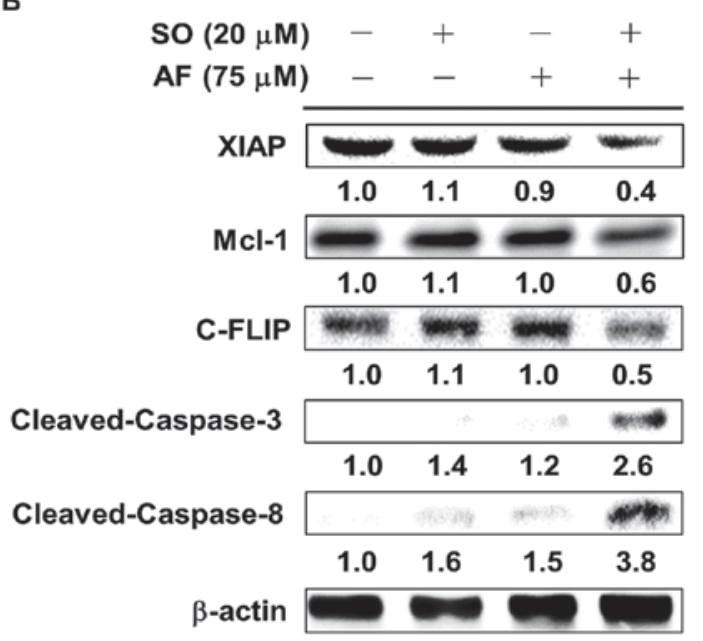

Cytochrome-C

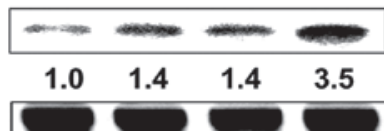

Figure 3. Effect of amentoflavone on sorafenib-induced expression of anti-apoptotic and pro-apoptotic proteins in SK-Hep1R cells. (A) SK-Hep1 cells were treated with $20 \mu \mathrm{M}$ sorafenib for $24 \mathrm{~h}$ and expression of anti-apoptotic proteins (XIAP, Mcl-1 and C-FLIP) was subsequently evaluated by western blot analysis. (B) SK-Hep1R cells were treated with $20 \mu \mathrm{M}$ sorafenib, $75 \mu \mathrm{M}$ amentoflavone or a combination of these for $24 \mathrm{~h}$. Expression of anti-apoptotic proteins and pro-apoptotic proteins (cleaved-Caspase-3, -8 and cytochrome $c$ ) was determined with western blot analysis. SK-Hep1R, SK-Hep1 sorafenib-resistant; XIAP, X-linked inhibitor of apoptosis protein; Mcl-1, myeloid cell leukemia-1; C-FLIP, cellular FADD-like IL-1 $\beta$ converting enzyme FLICE-like inhibitory protein; AF, amentoflavone; SO, sorafenib.

study. Initially, the differences in sorafenib-induced cytotoxicity and apoptosis between wild-type and sorafenib-resistant SK-Hep1 cells were investigated. SK-hep1R cells were resistant to sorafenib-induced cytotoxicity and apoptosis (Fig. 1A-C). Secondly, amentoflavone was revealed to enhance sorafenib-induced cytotoxicity and apoptosis in SK-hep1R cells (Fig. 2A-D). Finally, amentoflavone was demonstrated to inhibit expression of sorafenib-induced anti-apoptotic proteins (XIAP, Mcl-1 and C-FLIP), and triggered sorafenib-induced apoptosis through extrinsic and intrinsic pathways in SK-Hep1R cells (Fig. 3B).

Apoptosis is the process of programmed cell death, which may be triggered by extrinsic and intrinsic signal pathways. Apoptosis results in morphological change and DNA fragmentation, resulting in cell death (21). Various anticancer agents inhibit tumor growth through induction of apoptosis (22). Multiple anti-apoptotic proteins, including C-FLIP, XIAP and Mcl-1, are induced and overexpressed by anticancer agents and subsequently block apoptotic pathways (23). Caspase- 8 is a critical mediator of the extrinsic apoptotic pathway. C-FLIP disrupts initiation of extrinsic apoptotic pathway through inhibition of Caspase- 8 activation (21). The intrinsic apoptosis pathway is characterized by loss of mitochondrial membrane potential and release of cytochrome $c$. Mcl-1 inhibits the intrinsic apoptosis pathway by preventing loss of mitochondrial membrane potential and the release of cytochrome $c(24,25)$. A previous study indicated that sorafenib enhances vorinostat-induced extrinsic and intrinsic apoptotic pathways via inhibiting expression

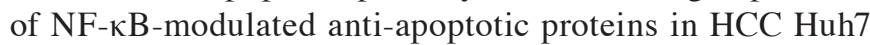
cells in vitro and in vivo (4). The present study also revealed that sorafenib induced accumulation of the $\mathrm{subG}_{1}$ population and loss of MMP, and inhibited protein levels of XIAP, Mcl-1 and C-FLIP in wild-type SK-Hep1 cells (Figs. 1B, C and $3 \mathrm{~A})$.

Apoptosis is inhibited and anti-apoptotic proteins are overexpressed in $\mathrm{HCC}$ cells with acquired resistance to sorafenib (8-10). Tai et al (9) reported that protein levels of activated Cyclin D1, Mcl-1 and STAT-3 in sorafenib-resistant HCC cells were increased compared with those in wild-type

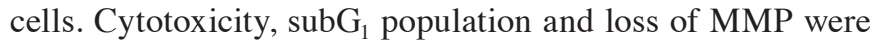
increased in SK-Hep1 cells compared with in SK-Hep1R cells following treatment with $20 \mu \mathrm{M}$ sorafenib for $24 \mathrm{~h}$ (Fig. 2B and C). Protein levels of XIAP, Mcl-1 and C-FLIP were not decreased by sorafenib treatment in SK-Hep1R cells (Fig. 3B). Hsu et al (10) suggested that Mcl-1 suppression is critical to restore sorafenib-induced apoptosis in sorafenib-resistant HCC cells. The present results revealed that amentoflavone not only decreased sorafenib-induced anti-apoptotic protein levels (XIAP, Mcl-1 and C-FLIP) but also triggered sorafenib-induced pro-apoptotic protein expression (cleaved-Caspase-3, -8 and cytochrome $c$ ) in SK-Hep1R cells (Fig. 3B). Notably, amentoflavone alone did not induce apoptosis but enhanced sorafenib-induced increases in the subG $_{1}$ population, loss of MMP and DNA fragmentation. Inhibition of sorafenib-induced protein levels of XIAP, Mcl-1 and C-FLIP by amentoflavone was associated with enhancement of sorafenib-induced apoptosis in SK-Hep1R cells. In conclusion, it was hypothesized that amentoflavone enhanced sorafenib-induced apoptosis through extrinsic and intrinsic pathways in SK-Hep1R cells. Application of amentoflavone as a sorafenib sensitizer may help to enhance the therapeutic efficacy of sorafenib in patients with HCC.

\section{Acknowledgements}

The present study was supported by the Taipei Medical University/Taipei Medical University Hospital (grant no. 104TMU-TMUH-23), the Yilan National Yang-Ming University Hospital (grant no. RD2016-022) Taipei Cathay General Hospital (grant no. CGH-MR-A10407) and Central Taiwan University of Science and Technology (grant no. CTU105-P-16). The authors would like to thank Professor Song-Shei Lin of the Department of Radiological Technology, Central Taiwan University of Science and Technology (Taichung, Taiwan) for technical assistance. The authors would like 
to thank the Translational Laboratory, Department of Medical Research, Taipei Medical University Hospital for their support.

\section{References}

1. Lencioni R, Llovet JM, Han G, Tak WY, Yang J, Guglielmi A, Paik SW, Reig M, Kim DY, Chau GY, et al: Sorafenib or placebo plus TACE with doxorubicin-eluting beads for intermediate stage HCC: The SPACE trial. J Hepatol 64: 1090-1098, 2016.

2. Liu L, Cao Y, Chen C, Zhang X, McNabola A, Wilkie D, Wilhelm S, Lynch $M$ and Carter C: Sorafenib blocks the $\mathrm{RAF} / \mathrm{MEK} / \mathrm{ERK}$ pathway, inhibits tumor angiogenesis, and induces tumor cell apoptosis in hepatocellular carcinoma model PLC/PRF/5. Cancer Res 66: 11851-11858, 2006.

3. Wilhelm SM, Adnane L, Newell P, Villanueva A, Llovet JM and Lynch M: Preclinical overview of sorafenib, a multikinase inhibitor that targets both Raf and VEGF and PDGF receptortyrosine kinase signaling. Mol Cancer Ther 7: 3129-3140, 2008.

4. Hsu FT, Liu YC, Chiang IT, Liu RS, Wang HE, Lin WJ and Hwang JJ: Sorafenib increases efficacy of vorinostat against human hepatocellular carcinoma through transductioninhibition of vorinostat-induced ERK/NF- $\mathrm{EB}$ signaling. Int J Oncol 45: 177-188, 2014

5. Huang CY, Lin CS, Tai WT, Hsieh CY, Shiau CW, Cheng AL and Chen KF: Sorafenib enhances radiation-induced apoptosis in hepatocellular carcinoma by inhibiting STAT3. Int J Radiat Oncol Biol Phys 86: 456-462, 2013.

6. van Malenstein H, Dekervel J, Verslype C, Van Cutsem E, Windmolders P, Nevens F and van Pelt J: Long-term exposure to sorafenib of liver cancer cells induces resistance with epithelial-to-mesenchymal transition, increased invasion and risk of rebound growth. Cancer Lett 329: 74-83, 2013.

7. Wörns MA, Schuchmann M, Düber C, Otto G, Galle PR and Weinmann A: Sunitinib in patients with advanced hepatocellular carcinoma after progression under sorafenib treatment. Oncology 79: 85-92, 2010.

8. Chen KF, Chen HL, Tai WT, Feng WC, Hsu CH, Chen PJ and Cheng AL: Activation of phosphatidylinositol 3-kinase/Akt signaling pathway mediates acquired resistance to sorafenib in hepatocellular carcinoma cells. J Pharmacol Exp Ther 337: 155-161, 2011.

9. Tai WT, Cheng AL, Shiau CW, Liu CY, Ko CH, Lin MW, Chen PJ and Chen KF: Dovitinib induces apoptosis and overcomes sorafenib resistance in hepatocellular carcinoma through SHP-1-mediated inhibition of STAT3. Mol Cancer Ther 11: 452-363, 2012.

10. Hsu C, Lin LI, Cheng YC, Feng ZR, Shao YY, Cheng AL and Ou DL: Cyclin E1 inhibition can overcome sorafenib resistance in hepatocellular carcinoma cells through Mcl-1suppression. Clin Cancer Res 22: 2555-2564, 2016.

11. Guruvayoorappan C and Kuttan G: Effect of amentoflavone on the inhibition of pulmonary metastasis induced by B16F-10 melanoma cells in C57BL/6 mice. Integr Cancer Ther 6: 185-197, 2007.
12. Chen JH, Chen WL and Liu YC: Amentoflavone induces anti-angiogenic and anti-metastatic effects through suppression of NF- $\mathrm{KB}$ activation in MCF-7 cells. Anticancer Res 35: 6685-6693, 2015.

13. Pei JS, Liu CC, Hsu YN, Lin LL, Wang SC, Chung JG, Bau DT and Lin SS: Amentoflavone induces cell-cycle arrest and apoptosis in MCF-7 human breast cancer cells via mitochondria-dependent pathway. In Vivo 26: 963-970, 2012.

14. Lee JS, Sul JY, Park JB, Lee MS, Cha EY, Song IS, Kim JR and Chang ES: Fatty acid synthase inhibition by amentoflavone suppresses HER2/neu (erbB2) oncogene in SKBR3 human breast cancer cells. Phytother Res 27: 713-720, 2013.

15. Lee S, Kim H, Kang JW, Kim JH, Lee DH, Kim MS, Yang Y, Woo ER, Kim YM, Hong J and Yoon DY: The biflavonoid amentoflavone induces apoptosis via suppressing E7 expression, cell cycle arrest at sub-G1 phase, and mitochondria-emanated intrinsic pathways in human cervical cancer cells. J Med Food 14: 808-816, 2011.

16. Ma CY,Ji WT, Chueh FS, Yang JS, Chen PY, Yu CC and Chung JG: Butein inhibits the migration and invasion of SK-HEP-1 human hepatocarcinoma cells through suppressing the ERK, JNK, p38, and uPA signaling multiple pathways. J Agric Food Chem 59: 9032-9038, 2011.

17. Zhai B, Hu F, Yan H, Zhao D, Jin X, Fang T, Pan S, Sun X and $\mathrm{Xu}$ L: Bufalin reverses resistance to sorafenib by inhibiting Akt activation in hepatocellular carcinoma: The role of endoplasmic reticulum stress. PLoS One 10: e0138485, 2015.

18. Wang WH, Chiang IT, Ding K, Chung JG, Lin WJ, Lin SS and Hwang JJ: Curcumin-induced apoptosis in human hepatocellular carcinoma j5 cells: Critical role of $\mathrm{ca}(+2)$-dependent pathway. Evid Based Complement Alternat Med 2012: 512907, 2012.

19. Huang SH, Wu LW, Huang AC, Yu CC, Lien JC, Huang YP, Yang JS, Yang JH, Hsiao YP, Wood WG, et al: Benzyl isothiocyanate (BITC) induces G2/M phase arrest and apoptosis in human melanoma A375.S2 cells through reactive oxygen species (ROS) and both mitochondria-dependent and death receptor-mediated multiple signaling pathways. J Agric Food Chem 60: 665-675, 2012.

20. Ting CY, Wang HE, Yu CC, Liu HC, Liu YC and Chiang IT: Curcumin triggers DNA damage and inhibits expression of DNA repair proteins in human lung cancer cells. Anticancer Res 35: 3867-3873, 2015.

21. Elmore S: Apoptosis: A review of programmed cell death. Toxicol Pathol 35: 495-516, 2007.

22. Johnstone RW, Ruefli AA and Lowe SW: Apoptosis: A link between cancer genetics and chemotherapy. Cell 108: 153-164, 2002.

23. Igney FH and Krammer PH: Death and anti-death: Tumour resistance to apoptosis. Nat Rev Cancer 2: 277-288, 2002.

24. Perciavalle RM and Opferman JT: Delving deeper: MCL-1's contributions to normal and cancer biology. Trends Cell Biol 23: 22-29, 2013.

25. Morciano G, Giorgi C, Balestra D, Marchi S, Perrone D, Pinotti M and Pinton P: Mcl-1 involvement in mitochondrial dynamics is associated with apoptotic cell death. Mol Biol Cell 27: 20-34, 2016. 